\title{
The Numerical Solution of Linear Sixth Order Boundary Value Problems with Quartic B-Splines
}

\author{
Mingzhu Li, ${ }^{1,2}$ Lijuan Chen, ${ }^{1}$ and Qiang $\mathrm{Ma}^{2}$ \\ ${ }^{1}$ School of Science, Qingdao Technological University, Qingdao 266033, China \\ ${ }^{2}$ Department of Mathematics, Harbin Institute of Technology at Weihai, Weihai 264209, China \\ Correspondence should be addressed to Qiang Ma; hitmaqiang@hotmail.com
}

Received 8 September 2013; Accepted 16 November 2013

Academic Editor: Luís Godinho

Copyright (c) 2013 Mingzhu Li et al. This is an open access article distributed under the Creative Commons Attribution License, which permits unrestricted use, distribution, and reproduction in any medium, provided the original work is properly cited.

\begin{abstract}
A quartic B-spline method is proposed for solving the linear sixth order boundary value problems. The method converts the boundary problem to solve a system of linear equations and obtains coefficients of the corresponding B-spline functions. The method has the convergence of two order. It develops not only the quartic spline approximate solution but also the higher order approximate derivatives. Two numerical examples are presented to verify the theoretical analysis and show the validity and applicability of the method. Compared with other existing recent methods, the quartic B-spline method is a more efficient and effective tool.
\end{abstract}

\section{Introduction}

In the paper, we consider the linear sixth order boundary value problems (6BVP) of the form:

$$
y^{(6)}(x)+f(x) y(x)=g(x),
$$

subject to the conditions

$$
\begin{aligned}
& y(a)=A_{0}, \quad y^{\prime}(a)=A_{1}, \quad y^{\prime \prime}(a)=A_{2}, \\
& y(b)=B_{0}, \quad y^{\prime}(b)=B_{1}, \quad y^{\prime \prime}(b)=B_{2},
\end{aligned}
$$

where $f(x)$ and $g(x)$ are continuous functions on $[a, b]$, and[? ] $A_{i}(i=0,1,2)$ and $B_{i}(i=0,1,2)$ are given finite real constants. Many mathematical models arising in various applications can be written as boundary value problems. One such problem is the sixth boundary value problem which plays an important role in astrophysics and the narrow convecting layers bounded by stable layers [1-4]. Further discussion of sixth order boundary value problems is given in $[5,6]$ and in a book by Chandrasekhar [7]. Theorems that list conditions for the existence and uniqueness of solution of such type of boundary value problems can be found in the book written by Agarwal [8]. However, it is difficult to obtain the analytic solutions of (1)-(2). Therefore, the availability of numerical method for this problem is of practical importance.

Over the years, there are several authors who worked on this type of boundary value problems by using different methods. For example, finite difference method was developed by Boutayeb and Twizell $[2,3,9]$. A modified form of the decomposition method was established by Wazwaz [10] and used to solve such BVPs [11]. Sinc-Galerkin method, variational iteration method, and homotopy perturbation method were developed to study the same problem [12-14]. Spline functions have been also used to construct efficient and accurate numerical methods for solving boundary value problems. For example, Siddiqi and Akram solved the same boundary value problems by using different splines such as quintic splines, septic splines, and nonpolynomial splines [15-17]. Loghmani and Viswanadham used sixth and septic $\mathrm{B}$-spline functions to solve sixth order boundary value problems $[18,19]$. Instead of the above two ways, other differential spline collocation methods can also be used [20-22].

It is well known that the quartic B-spline has been widely applied for the approximation solution of boundary value problems. Caglar used quartic B-spline to solve the linear cubic order boundary value problem [23]. Besides, quartic 
B-spline was also used to solve fifth order boundary value problems and the obstacle problems [24, 25]. Our method is based on quartic B-spline interpolation. It is second order convergent and with lower computational cost. Moreover, we also can get the approximate derivative values of $y^{(k)}(x)(k=$ $1,2,3,4,5)$ at the knots. This is another advantage of our method, since some methods cannot obtain those results.

This paper is arranged as follows. In Section 2, the definition of quintic B-splines has been described and some preliminary results of quartic B-spline interpolation have been presented. In Section 3, we mainly give the quartic Bspline solution of linear sixth-order boundary value problems based on the results. In Section 4, the convergence of the method has been demonstrated. In Section 5, numerical examples of linear boundary value problems are presented, which illustrate the performance of this method.

\section{Quartic B-Spline}

2.1. Definition of Quartic B-Spline. For an interval $[a, b] \subset R$, we introduce a set of equally spaced knots of partition $\Omega=$ $\left\{x_{0}, x_{1}, \ldots, x_{n}\right\}$, and we assume that $n \geq 5, x_{i}=a+i h(i=$ $0,1, \ldots, n), x_{0}=a, x_{n}=b$.

Let $S_{4}[\pi]$ be the space of continuously differentiable, piecewise, quartic-degree polynomials on $\pi$. Consider the B-splines basis in $S_{4}[\pi]$. A detailed description of B-spline functions generated by subdivision can be founded in [26].

The zero degree B-spline is defined as

$$
N_{i, 0}(x)= \begin{cases}1, & x \in\left[x_{i}, x_{i+1}\right], \\ 0, & \text { otherwise },\end{cases}
$$

and for positive constant $p$, it is defined in the following recursive form:

$$
\begin{array}{r}
N_{i, p}(x)=\frac{x-x_{i}}{x_{i+p}-x_{i}} N_{i, p-1}(x)+\frac{x_{i+p+1}-x}{x_{i+p+1}-x_{i+1}} N_{i+1, p-1}, \\
p \geq 2 .
\end{array}
$$

We apply this recursion to get the quartic B-spline $N_{i, 4}(x)(i=-2,-1, \ldots, n+1)$; it is defined in $S_{4}[\pi]$ as follows:

$$
N_{i, 4}(x)
$$

$$
=\frac{1}{24 h^{4}} \begin{cases}\left(x-x_{i-2}\right)^{4}, & x \in\left[x_{i-2}, x_{i-1}\right], \\ \left(x-x_{i-2}\right)^{4}-5\left(x-x_{i-1}^{4}\right), & x \in\left[x_{i-1}, x_{i}\right], \\ \left(x-x_{i-2}\right)^{4}-5\left(x-x_{i-1}\right)^{4} & \\ +10\left(x-x_{i}\right)^{4}, & x \in\left[x_{i}, x_{i+1}\right], \\ \left(x-x_{i+3}\right)^{4}-5\left(x-x_{i+2}\right)^{4}, & x \in\left[x_{i+1}, x_{i+2}\right], \\ \left(x-x_{i+3}\right)^{4}, & x \in\left[x_{i+2}, x_{i+3}\right], \\ 0, & \text { otherwise. }\end{cases}
$$

The properties of quartic B-spline functions:

(1) compact supported:

$$
N_{i, 4}(x)= \begin{cases}\geq 0, & x \in\left[x_{i-2}, x_{i+3}\right], \\ =0, & \text { otherwise. }\end{cases}
$$

TABLE 1: The values of $N_{i, 4}^{(k)}(x)$ at the knots.

\begin{tabular}{cccccc}
\hline & $x_{i-1}$ & $x_{i}$ & $x_{i+1}$ & $x_{i+2}$ & Otherwise \\
\hline$N_{i, 4}(x)$ & $1 / 24$ & $11 / 24$ & $11 / 24$ & $1 / 24$ & 0 \\
$N_{i, 4}^{\prime}(x)$ & $1 / 6 h$ & $3 / 6 h$ & $-3 / 6 h$ & $-1 / 6 h$ & 0 \\
$N_{i, 4}^{\prime \prime}(x)$ & $1 / 2 h^{2}$ & $-1 / 2 h^{2}$ & $-1 / 2 h^{2}$ & $1 / 2 h^{2}$ & 0 \\
$N_{i, 4}^{\prime \prime \prime}(x)$ & $1 / h^{3}$ & $-3 / h^{3}$ & $3 / h^{3}$ & $-1 / h^{3}$ & 0 \\
\hline
\end{tabular}

(2) Normalization: $\sum_{i=-2}^{n+1} N_{i, 4}(x)=1$.

(3) Translation invariance: $N_{i, 4}(x)=N_{0,4}(x-(i-1) h)(i=$ $-2,-1, \ldots, n+1)$.

(4) Derivation formula: $N_{i, 4}^{(k)}(x)=(4 ! /(4-$ k)!) $\sum_{j=1}^{n} \alpha_{k, j} N_{i+j, 4-k}$, where

$$
\begin{gathered}
\alpha_{0,0}=1, \\
\alpha_{k, 0}=\frac{\alpha_{k-1,0}}{x_{i+3-k}-x_{i}}, \\
\alpha_{k, k}=\frac{-\alpha_{k-1, k-1}}{x_{i+5}-x_{i+k}}, \\
\alpha_{k, j}=\frac{\alpha_{k-j, j}-\alpha_{k-1, j-1}}{x_{i+j+5-k}-x_{i+j}} .
\end{gathered}
$$

By some trivial computations, we can obtain the value of $N_{i, 4}^{(k)}(x)(i=-2,-1, \ldots, n+1, k=0,1,2,3)$ at the knots, which are listed in Table 1.

2.2. Quartic B-Spline Interpolation. For a given function $y(x)$ (assuming to be sufficiently smooth), there exists a unique quartic B-spline $s(x)=\sum_{i=-2}^{i=n+1} c_{i} N_{i, 4}(x)$ satisfying the interpolation conditions

$$
\begin{array}{ll}
s\left(x_{i}\right)=y\left(x_{i}\right), & (i=0,1, \ldots, n), \\
s^{\prime}(a)=y^{\prime}(a), & s^{\prime \prime}(a)=y^{\prime \prime}(a), \\
s^{\prime}(b)=y^{\prime}(b), & s^{\prime \prime}(b)=y^{\prime \prime}(b) .
\end{array}
$$

For $j=0,1, \ldots, n$, let $y_{j}=s\left(x_{j}\right)=y\left(x_{j}\right), P_{j}=s^{\prime}\left(x_{j}\right)$, $Q_{j}=s^{\prime \prime}\left(x_{j}\right)$, and $R_{j}=s^{(3)}\left(x_{j}\right)$ for short. Through a simple calculation by Table 1 , we have

$$
\begin{aligned}
y_{j} & =\sum_{i=-2}^{n+1} c_{i} N_{i, 4}\left(x_{j}\right)=\frac{1}{24}\left(c_{j-2}+11 c_{j-1}+11 c_{j}+c_{j+1}\right), \\
P_{j} & =\sum_{i=-2}^{n+1} c_{i} N_{i, 4}^{\prime}\left(x_{j}\right)=\frac{1}{6 h}\left(-c_{j-2}-3 c_{j-1}+3 c_{j}+c_{j+1}\right), \\
Q_{j} & =\sum_{i=-2}^{n+1} c_{i} N_{i, 4}^{\prime \prime}\left(x_{j}\right)=\frac{1}{2 h^{2}}\left(c_{j-2}-c_{j-1}-c_{j}+c_{j+1}\right), \\
R_{j} & =\sum_{i=-2}^{n+1} c_{i} N_{i, 4}^{\prime \prime \prime}\left(x_{j}\right)=\frac{1}{h^{3}}\left(-c_{j-2}+3 c_{j-1}-3 c_{j}+c_{j+1}\right) .
\end{aligned}
$$


So, we get

$$
\begin{gathered}
\frac{h}{4}\left(P_{j-2}+11 P_{j-1}+11 P_{j}+P_{j+1}\right) \\
=-y_{j-2}-3 y_{j-1}+3 y_{j}+y_{j+1}, \\
\frac{h^{2}}{12}\left(Q_{j-1}+10 Q_{j}+Q_{j+1}\right)=y_{j-1}-2 y_{j}+y_{j+1}, \\
\frac{h^{3}}{24}\left(R_{j-2}+11 R_{j-1}+11 R_{j}+R_{j+1}\right) \\
=-y_{j-2}+3 y_{j-1}-3 y_{j}+y_{j+1} .
\end{gathered}
$$

Using operator notation in $[27,28]$, we obtain

$$
\begin{gathered}
P_{j}=\frac{4}{h}\left(\frac{-E^{-2}-3 E^{-1}+3 I+E}{E^{-2}+11 E^{-1}+11 I+E}\right) y_{j}, \\
Q_{j}=\frac{12}{h^{2}}\left(\frac{E^{-1}-2 I+E}{E^{-1}+10 I+E}\right) y_{j}, \\
R_{j}=\frac{24}{h^{3}}\left(\frac{-E^{-2}+3 E^{-1}-3 I+E}{E^{-2}+11 E^{-1}+11 I+E}\right) y_{j},
\end{gathered}
$$

where the operators are defined $E y(x)=y(x+h), D y(x)=$ $y^{\prime}(x)$, and $I y(x)=y(x)$. Let $E=\exp (h D)$ and expand them in powers of $h D$; we get

$$
\begin{gathered}
y^{\prime}\left(x_{j}\right)=P_{j}-\frac{1}{720} h^{4} y^{(5)}\left(x_{j}\right)+O\left(h^{6}\right), \\
y^{\prime \prime}\left(x_{j}\right)=Q_{j}+\frac{1}{240} h^{4} y^{(6)}\left(x_{j}\right)+O\left(h^{6}\right), \\
y^{\prime \prime \prime}\left(x_{j}\right)=R_{j}-\frac{1}{12} h^{2} y^{(5)}\left(x_{j}\right)+\frac{1}{240} h^{4} y^{(7)}\left(x_{j}\right)+O\left(h^{6}\right) .
\end{gathered}
$$

We can use $R_{j}$ to construct numerical difference formula for $y^{(4)}\left(x_{j}\right), y^{(5)}\left(x_{j}\right)$, and $y^{(6)}\left(x_{j}\right)(j=1,2, \ldots, n-1)$ by the Taylor series expansion as follows:

$$
\begin{gathered}
y^{(4)}\left(x_{j}\right)=\frac{R_{j+1}-R_{j-1}}{2 h}+O\left(h^{2}\right), \\
y^{(5)}\left(x_{j}\right)=\frac{R_{j+1}-2 R_{j}+R_{j-1}}{h^{2}}+O\left(h^{2}\right), \\
y^{(6)}\left(x_{j}\right)=\left(\left(\frac{R_{j+2}-R_{j}}{2 h}-2 \frac{R_{j+1}-R_{j-1}}{2 h}\right.\right. \\
\left.\left.+\frac{R_{j}-R_{j-2}}{2 h}\right) \times\left(h^{2}\right)^{-1}\right)+O\left(h^{2}\right), \\
=\frac{R_{j+2}-2 R_{j+1}+2 R_{j-1}-R_{j-2}}{2 h^{3}}+O\left(h^{2}\right) .
\end{gathered}
$$

Substituting (9)-(12) into (15)-(18) yields

$$
\begin{gathered}
y^{\prime}\left(x_{j}\right)=\frac{1}{6 h}\left(-c_{j-2}-3 c_{j-1}+3 c_{j}+c_{j+1}\right)+O\left(h^{4}\right), \\
y^{\prime \prime}\left(x_{j}\right)=\frac{1}{2 h^{2}}\left(c_{j-2}-c_{j-1}-c_{j}+c_{j+1}\right)+O\left(h^{4}\right), \\
y^{\prime \prime \prime}\left(x_{j}\right)=\frac{1}{h^{3}}\left(-c_{j-2}+3 c_{j-1}-3 c_{j}+c_{j+1}\right)+O\left(h^{2}\right), \\
y^{(4)}\left(x_{j}\right)=\frac{1}{2 h^{4}}\left(c_{j-3}-3 c_{j-2}+2 c_{j-1}+2 c_{j}\right. \\
\left.\quad-3 c_{j+1}+c_{j+2}\right)+O\left(h^{2}\right), \\
y^{(5)}\left(x_{j}\right)=\frac{1}{h^{5}}\left(-c_{j-3}+5 c_{j-2}-10 c_{j-1}+10 c_{j}\right. \\
\left.-5 c_{j+1}+c_{j+2}\right)+O\left(h^{4}\right), \\
y^{(6)}\left(x_{j}\right)=\frac{1}{2 h^{6}}\left(c_{j-4}-5 c_{j-3}+9 c_{j-2}-5 c_{j-1}-5 c_{j}\right. \\
\left.+9 c_{j+1}-5 c_{j+2}+c_{j+3}\right)+O\left(h^{2}\right) .
\end{gathered}
$$

\section{Description of Numerical Method}

In the section, we give the quartic B-spline method for the linear sixth order boundary value problem. Let $s(x)=$ $\sum_{i=-2}^{n+1} c_{i} N_{i, 4}(x)$ be the approximate solution of 6BVP (1)-(2) and $\widetilde{s(x)}=\sum_{i=-2}^{n+1} \widetilde{c}_{i} N_{i, 4}(x)$ the approximate spline of $s(x)$. Discretize (1) at the knots $x_{i}(i=2,3, \ldots, n-2)$, we get

$$
y^{(6)}\left(x_{i}\right)+f\left(x_{i}\right) y\left(x_{i}\right)=g\left(x_{i}\right) .
$$

By (9) and (18), we turn (20) into

$$
\begin{aligned}
& \frac{1}{2 h^{6}}\left(c_{i-4}-5 c_{i-3}+9 c_{i-2}-5 c_{i-1}\right. \\
& \left.-5 c_{i}+9 c_{i+1}-5 c_{i+2}+c_{i+3}\right)+\frac{f\left(x_{i}\right)}{24} \\
& \times\left(c_{i-2}+11 c_{i-1}+11 c_{i}+c_{i+1}\right)=g\left(x_{i}\right)+O\left(h^{2}\right),
\end{aligned}
$$

where $f\left(x_{i}\right)$ and $g\left(x_{i}\right)$ are the value of $f(x)$ and $g(x)$ at the knots $x_{i}(i=2,3, \ldots, n-2)$ for short. Change (21) equivalently, we yield

$$
\begin{aligned}
12 & \left(c_{i-4}-5 c_{i-3}+9 c_{i-2}-5 c_{i-1}-5 c_{i}\right. \\
& \left.+9 c_{i+1}-5 c_{i+2}+c_{i+3}\right)+f\left(x_{i}\right) \\
& \times\left(c_{i-2}+11 c_{i-1}+11 c_{i}+c_{i+1}\right) h^{6} \\
= & 24 h^{6} g\left(x_{i}\right)+O\left(h^{8}\right) .
\end{aligned}
$$


Dropping the term $O\left(h^{8}\right)$ from (22), we get a linear system with $n-3$ linear equations in $n+4$ unknowns $c_{i}(i=$ $-2,-1, \ldots, n+1)$

$$
\begin{aligned}
& 12\left(c_{i-4}-5 c_{i-3}+9 c_{i-2}-5 c_{i-1}-5 c_{i}\right. \\
& \left.+9 c_{i+1}-5 c_{i+2}+c_{i+3}\right)+f\left(x_{i}\right) \\
& \quad \times\left(c_{i-2}+11 c_{i-1}+11 c_{i}+c_{i+1}\right) h^{6} \\
& =24 h^{6} g\left(x_{i}\right) \quad(i=2,3, \ldots, n-2),
\end{aligned}
$$

so seven more equations are needed.

By the boundary conditions at $x=a$

$$
\begin{gathered}
y(a)=A_{0}, \\
y^{\prime}(a)=A_{1}, \\
y^{\prime \prime}(a)=A_{2},
\end{gathered}
$$

we have

$$
\begin{gathered}
c_{-2}+11 c_{-1}+11 c_{0}+c_{1}=24 A_{0} \\
-c_{-2}-3 c_{-1}+3 c_{0}+c_{1}=6 h A_{1}, \\
c_{-2}-c_{-1}-c_{0}+c_{1}=2 h^{2} A_{2} .
\end{gathered}
$$

By the boundary conditions at $x=b$

$$
\begin{gathered}
y(b)=B_{0}, \\
y^{\prime}(b)=B_{1}, \\
y^{\prime \prime}(b)=B_{2},
\end{gathered}
$$

we get

$$
\begin{gathered}
c_{n-2}+11 c_{n-1}+11 c_{n}+c_{n+1}=24 B_{0}, \\
-c_{n-2}-3 c_{n-1}+3 c_{n}+c_{n+1}=6 h B_{1}, \\
c_{n-2}-c_{n-1}-c_{n}+c_{n+1}=2 h^{2} B_{2} .
\end{gathered}
$$

We can construct an approximate formula as follows:

$$
y^{(6)}(a)=\frac{-5 R_{0}+18 R_{1}-24 R_{2}+14 R_{3}-3 R_{4}}{24 h^{3}}+O\left(h^{2}\right) \text {, }
$$

where the coefficients are determined by maximizing the error order.

Substituting (12) into (28), we have

$$
\begin{aligned}
& 5 c_{-2}-33 c_{-1}+93 c_{0}-145 c_{1}+135 c_{2} \\
& \quad-75 c_{3}+23 c_{4}-3 c_{5}=24 h^{6} y^{(6)}(a)+O\left(h^{5}\right) .
\end{aligned}
$$

Dropping the term $O\left(h^{5}\right)$ from (29), we can get

$$
\begin{aligned}
& 5 c_{-2}-33 c_{-1}+93 c_{0}-145 c_{1}+135 c_{2}-75 c_{3} \\
& +23 c_{4}-3 c_{5}=24 h^{6} y^{(6)}(a) .
\end{aligned}
$$

Take (22), (25), (27), and (29) together, we get $n+4$ linear equation with $c_{i}(i=-2,-1, \ldots, n+1)$ as unknowns. The linear system can be written in matrix notations

$$
\left(A+h^{6} F B\right) C=D+E .
$$

Take (23), (25), (27), and (30) together, we get $n+4$ linear equation with $\widetilde{c}_{i}(i=-2,-1, \ldots, n+1)$ as unknowns. The linear system can be written in matrix notations

$$
\left(A+h^{6} F B\right) \widetilde{C}=D
$$

where

$$
\begin{gathered}
C=\left(c_{-2}, c_{-1}, c_{0}, \ldots, c_{n+1}\right)^{T}, \\
\widetilde{C}=\left(\widetilde{c}_{-2}, \widetilde{c}_{-1}, \widetilde{c}_{0}, \ldots, \widetilde{c}_{n+1}\right)^{T}, \\
D=\left(24 h^{6} y^{(6)}(a), 24 A_{0}, 6 h A_{1}, 2 h^{2} A_{2}, 24 h^{6} g\left(x_{2}\right), \ldots,\right. \\
\left.24 h^{6} g\left(x_{n-1}\right), 24 B_{0}, 6 h B_{1}, 2 h^{2} B_{2}\right)^{T}, \\
E=\left(e_{-2}, e_{-1}, \ldots, e_{n+1}\right)^{T}, \\
e_{-1}=e_{0}=e_{1}=e_{n-1}=e_{n}=e_{n+1}=0, \\
e_{-2}=O\left(h^{5}\right), \quad e_{i}=O\left(h^{8}\right) \quad(i=2, \ldots, n-2),
\end{gathered}
$$

A

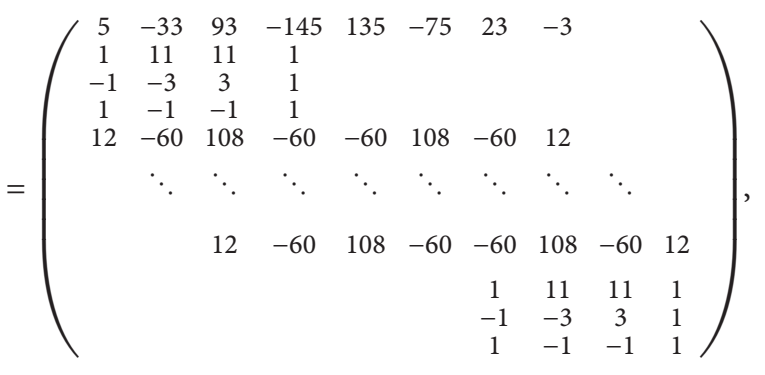

F

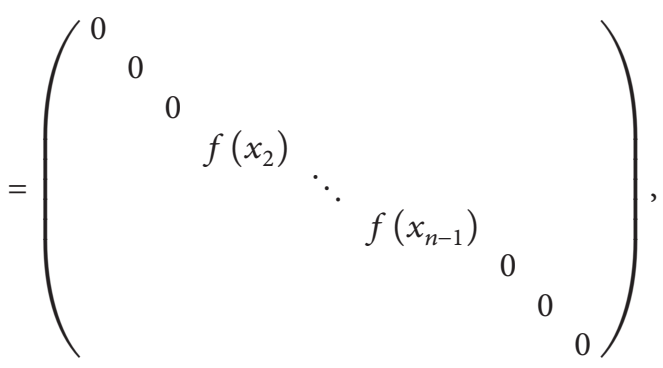


TABLE 2: $\operatorname{Max}\left[y^{(\nu)}\left(x_{i}\right)\right]$ of quartic B-spline method for Example 1.

\begin{tabular}{ccccccc}
\hline$n$ & $\max \left[y\left(x_{i}\right)\right]$ & $\max \left[y^{\prime}\left(x_{i}\right)\right]$ & $\max \left[y^{\prime \prime}\left(x_{i}\right)\right]$ & $\max \left[y^{\prime \prime \prime}\left(x_{i}\right)\right]$ & $\max \left[y^{(4)}\left(x_{i}\right)\right]$ & $\max \left[y^{(5)}\left(x_{i}\right)\right]$ \\
\hline 10 & $3.2599 e-6$ & $1.3642 e-5$ & $1.9072 e-4$ & $6.1038 e-3$ & $1.1021 e-2$ & $8.444 e-1$ \\
20 & $1.3846 e-7$ & $5.1383 e-7$ & $6.7388 e-6$ & $1.2107 e-5$ & $2.1304 e-4$ & $4.2045 e-2$ \\
40 & $2.8847 e-8$ & $1.5453 e-7$ & $1.3402 e-6$ & $2.8482 e-5$ & $7.4701 e-4$ & $2.1074 e-2$ \\
80 & $1.3493 e-8$ & $7.8389 e-8$ & $6.657 e-7$ & $7.5303 e-5$ & $3.1822 e-4$ & $1.602 e-2$ \\
\hline
\end{tabular}

$B$

$$
=\left(\begin{array}{cccccccccc}
0 & 0 & 0 & & & & & & \\
0 & 0 & 0 & & & & & & \\
0 & 0 & 0 & & & & & & \\
1 & 11 & 11 & 1 & 0 & 0 & 0 & & \\
& \ddots & \ddots & \ddots & \ddots & \ddots & \ddots & \ddots & \\
& & 0 & 0 & 0 & 1 & 11 & 11 & 1 \\
& & & & & & 0 & 0 & 0 \\
& & & & & & 0 & 0 & 0 \\
& & & & & & 0 & 0 & 0
\end{array}\right) .
$$

After solving the linear system (32), we obtain the quartic spline approximate solution $y(x) \approx \widetilde{s}(x)=\sum_{i=-2}^{n+1} \widetilde{c}_{i} N_{i, 4}(x)$. Furthermore, we can take $\widetilde{s}^{(k)}(x)(k=1,2,3,4,5)$ as the approximation of $y^{(k)}(x)(k=1,2,3,4,5)$.

\section{Convergence Analysis}

By (31) and (32), we have

$$
\left(A+h^{6} F B\right)(C-\widetilde{C})=E .
$$

$A$ is invertible, and if we assume that

$$
h^{6}\left\|A^{-1}\right\|_{\infty}\|F\|_{\infty}\|B\|_{\infty}<1 \text {, }
$$

then $\left(I+h^{8} A^{-1} F B\right)$ is also invertible.

Hence, we get

$$
C-\widetilde{C}=\left(I+h^{6} A^{-1} F B\right)^{-1} A^{-1} E .
$$

By (35) and (36) and note $\|E\|_{\infty} \leq k h^{8}$, we have

$$
\begin{aligned}
\|C-\widetilde{C}\|_{\infty} & \leq \frac{\left\|A^{-1}\right\|_{\infty}\|E\|_{\infty}}{1-h^{6}\left\|A^{-1}\right\|_{\infty}\|F\|_{\infty}\|B\|_{\infty}} \\
& \leq \frac{k h^{6}\left\|A^{-1}\right\|_{\infty}}{1-h^{6}\left\|A^{-1}\right\|_{\infty}\|B\|_{\infty}\|F\|_{\infty}} h^{2} \\
& \leq \frac{k}{\|B\|_{\infty}\|F\|_{\infty}} h^{2}=O\left(h^{2}\right) .
\end{aligned}
$$

Hence,

$$
\begin{aligned}
\|s-\widetilde{s}\|_{\infty} & \leq\|C-\widetilde{C}\|_{\infty} \sum_{i=1}^{n} N_{i, 4}(x) \\
& =\|C-\widetilde{C}\|_{\infty}=O\left(h^{2}\right) .
\end{aligned}
$$

Generally, we get

$$
\begin{aligned}
\|y-\widetilde{s}\|_{\infty} & \leq\|y-\widetilde{s}\|_{\infty}+\|s-\widetilde{s}\|_{\infty} \\
& =O\left(h^{2}\right)+O\left(h^{2}\right)=O\left(h^{2}\right) .
\end{aligned}
$$

\section{Numerical Results}

In the section, we give some computational results of numerical experiments with method based on previous sections to support our theoretical discussion. We use double precision arithmetic in order to reduce the round-off errors to a minimum.

Example 1. We consider the following equation:

$$
\begin{gathered}
y^{(6)}(x)+y(x)=6 \cos x, \quad 0 \leq x \leq 1, \\
y(0)=0, \quad y^{\prime}(0)=-1, \quad y^{\prime \prime}(0)=2, \\
y(1)=0, \quad y^{\prime}(1)=\sin 1, \quad y^{\prime \prime}(1)=2 \cos 1 .
\end{gathered}
$$

The exact solution is given by $y(x)=(x-1) \sin x$.

The results of maximum absolute errors $\max \left[y^{(\nu)}\left(x_{i}\right)\right]=$ $\max _{1 \leqslant i \leqslant n}\left|y^{(\nu)}\left(x_{i}\right)-s^{(\nu)}\left(x_{i}\right)\right|(\nu=0,1,2,3,4,5)$ for this problem are tabulated in Table 2.

Next, we compare our method with the other spline method. Consider another sixth order boundary value problem.

Example 2. Consider the boundary value problem

$$
\begin{aligned}
& y^{(6)}(x)-y(x)=-6 e^{x}, \quad 0 \leq x \leq 1, \\
& y(0)=1, \quad y^{\prime}(0)=0, \quad y^{\prime \prime}(0)=-1, \\
& y(1)=0, \quad y^{\prime}(1)=-e, \quad y^{\prime \prime}(1)=-2 e,
\end{aligned}
$$

which has the exact solution $y(x)=(1-x) e^{x}$.

The example has been solved by the collocation method based on the sixth B-spline [18], and the numerical results are stated in Table 3. Also, the system of differential equation along with the given boundary conditions was solved by Wazwaz using Adomian decomposition method [10] and Noor using variational iteration method [13]. The respective maximum absolute errors are given in Table 3. Obviously, the results of our method are very encouraging. 
TABle 3: $\operatorname{Max}\left[y\left(x_{i}\right)\right]$ of different methods for Example 2.

\begin{tabular}{lccccc}
\hline$x$ & Exact solution & {$[18]$} & {$[13]$} & {$[10]$} & Our method \\
\hline 0.1 & 0.99465383 & $1.2159 e-5$ & $4.0933 e-4$ & $4.0933 e-4$ & $7.5092 e-6$ \\
0.2 & 0.97712221 & $2.7418 e-5$ & $7.7820 e-4$ & $1.7820 e-4$ & $1.2619 e-5$ \\
0.3 & 0.94490117 & $2.2053 e-6$ & $1.0704 e-3$ & $1.2578 e-3$ & $1.94 e-3$ \\
0.4 & 0.89509482 & $2.5033 e-6$ & $1.2578 e-3$ & $1.2578 e-3$ & $2.1632 e-5$ \\
0.5 & 0.82436064 & $5.4836 e-6$ & $1.3223 e-3$ & $1.0704 e-3$ & $1.9704 e-5$ \\
0.6 & 0.72884752 & $1.6212 e-5$ & $1.2578 e-3$ & $7.7820 e-4$ & $8.2238 e-6$ \\
0.7 & 0.60412581 & $2.0682 e-5$ & $1.0704 e-3$ & $4.0933 e-4$ & $2.9420 e-6$ \\
0.8 & 0.44510819 & $2.2619 e-5$ & $7.7820 e-4$ & $4.0933 e-4$ & $2.3610 e-7$ \\
0.9 & 0.24596031 & $1.9460 e-5$ &
\end{tabular}

\section{Conclusion}

In the section, we employ the quartic B-spline for solving the sixth order boundary value problems. Properties of the B-spline function are utilized to reduce the computation of this problem to some algebraic equations. The method is computationally attractive and applications are demonstrated through illustrative examples. The obtained results showed that this approach can solve the problem effectively, and the comparison shows that the proposed technique is in good agreement with the existing results in the literature.

\section{References}

[1] J. Toomore, J. R. Zahn, and J. Latour, "Steller convection theory II: single-model study of the second convection zone in A-type starts," Astrophysical Journal, vol. 207, pp. 545-563, 1976.

[2] A. Boutayeb and E. H. Twizell, "Numerical methods for the solution of special sixth-order boundary value problems," International Joural of Computer Mathematics, vol. 45, no. 3-4, pp. 207-223, 1992.

[3] E. H. Twizell and A. Boutayeb, "Numerical methods for the solution of special and general sixth order boundary value problems, with applications to Bénard layer eigenvalue problems," Proceedings the Royal of Society A, vol. 431, no. 1883, pp. 433450, 1990.

[4] S. S. Siddiqi and E. H. Twizell, "Spline solutions of linear sixth-order boundary-value problems," International Journal of Computer Mathematics, vol. 60, no. 3-4, pp. 295-304, 1996.

[5] P. Bladwin, "Asymptotic estimates of the eigenvalues of a sixth order boundary value problem obtained by using global phaseintegral methods," Proceedings the Royal of Society A, vol. 322, no. 1566, pp. 281-305, 1987.

[6] P. Baldwin, "Localised instability in a Bénard layer," Applicable Analysis, vol. 24, no. 1-2, pp. 117-156, 1987.

[7] S. Chandrasekhar, Hydrodynamics and Hydromagentic Stability, New York, NY, USA, Dover edition, 1981.

[8] R. Agarwal, Boundary Value Problems for Higher Ordinary Differential Equations, World Scientific, Singapore, 1997.

[9] E. H. Twizell, Numerical Methods for Sixth-Order Boundary Value Problems, International Series of Numerical Mathematics, Numerical mathematics, Singapore, 1988.

[10] A. M. Wazwaz, "A reliable modification of Adomian decomposition method," Applied Mathematics and Computation, vol. 102, no. 1, pp. 77-86, 1999.
[11] A. M. Wazwaz, "The numerical solution of sixth-order boundary value problems by the modified decomposition method," Applied Mathematics and Computation, vol. 118, no. 2-3, pp. 311$325,2001$.

[12] M. El-Gamel, J. R. Cannon, and A. I. Zayed, "Sinc-Galerkin method for solving linear sixth-order boundary-value problems," Mathematics of Computation, vol. 73, no. 247, pp. 13251343, 2004.

[13] M. A. Noor, K. I. Noor, and S. T. Mohyud-Din, "Variational iteration method for solving sixth-order boundary value problems," Communications in Nonlinear Science and Numerical Simulation, vol. 14, no. 6, pp. 2571-2580, 2009.

[14] M. A. Noor and S. T. Mohyud-Din, "Homotopy perturbation method for solving sixth-order boundary value problems," Computers and Mathematics with Applications, vol. 55, no. 12, pp. 2953-2972, 2008.

[15] S. S. Siddiqi, G. Akram, and S. Nazeer, "Quintic spline solution of linear sixth-order boundary value problems," Applied Mathematics and Computation, vol. 189, no. 1, pp. 887-892, 2007.

[16] S. S. Siddiqi and G. Akram, "Septic spline solutions of sixthorder boundary value problems," Journal of Computational and Applied Mathematics, vol. 215, no. 1, pp. 288-301, 2008.

[17] G. Akram and S. S. Siddiqi, "Solution of sixth order boundary value problems using non-polynomial spline technique," Applied Mathematics and Computation, vol. 181, no. 1, pp. 708720, 2006.

[18] G. B. Loghmani and M. Ahmadinia, "Numerical solution of sixth order boundary value problems with sixth degree B-spline functions," Applied Mathematics and Computation, vol. 186, no. 2, pp. 992-999, 2007.

[19] K. S. K. Kasi Viswanadham and P. Murali Krishna, "Septic B-spline collocation method for sixth order boundary value problem," ARPN Journal of Engineering and Applied Sciences, vol. 5, no. 7, pp. 36-40, 2010.

[20] S. Siraj-ul-Islam, I. A. Tirmizi, F. Fazal-i-Haq, and M. A. Khan, "Non-polynomial splines approach to the solution of sixthorder boundary-value problems," Applied Mathematics and Computation, vol. 195, no. 1, pp. 270-284, 2008.

[21] M. A. Ramadan, I. F. Lashien, and W. K. Zahra, "A class of methods based on a septic non-polynomial spline function for the solution of sixth-order two-point boundary value problems," International Journal of Computer Mathematics, vol. 85, no. 5, pp. 759-770, 2008. 
[22] A. Lamnii, H. Mraoui, D. Sbibih, A. Tijini, and A. Zidna, "Spline collocation method for solving linear sixth-order boundaryvalue problems," International Journal of Computer Mathematics, vol. 85, no. 11, pp. 1673-1684, 2008.

[23] H. N. Caglar, S. H. Caglar, and E. H. Twizell, "Numerical solution of third-order boundary-value problems with fourthdegree B-spline functions," International Journal of Computer Mathematics, vol. 71, no. 3, pp. 373-381, 1999.

[24] F. G. Lang and X. P. Xu, "Quartic B-spline collocation method for fifth order boundary value problems," Computing, vol. 92, no. 4, pp. 365-378, 2011.

[25] F. Gao and C. Chi, "Solving third-order obstacle problems with quartic B-splines," Applied Mathematics and Computation, vol. 180, no. 1, pp. 270-274, 2006.

[26] C. De Boor, A Practice Guide Spline, Springer, New York, NY, USA, 1978.

[27] D. J. Fyfe, "The use of cubic splines in the solution of two-point boundary value problems," The Computer Journal, vol. 12, no. 2 , pp. 188-192, 1969.

[28] T. R. Lucas, "Error bounds for interpolating cubic splines under various end conditions," SIAM Journal on Numerical Analysis, vol. 11, no. 3, pp. 569-584, 1974. 


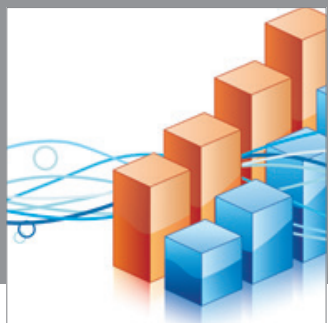

Advances in

Operations Research

mansans

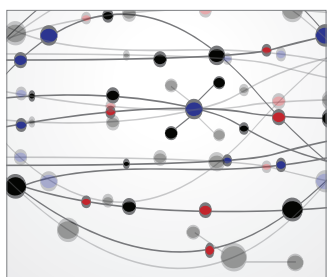

The Scientific World Journal
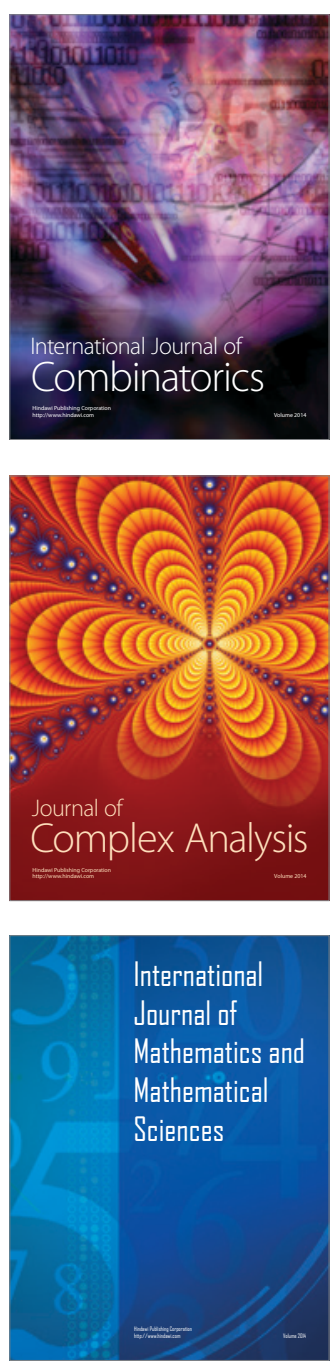
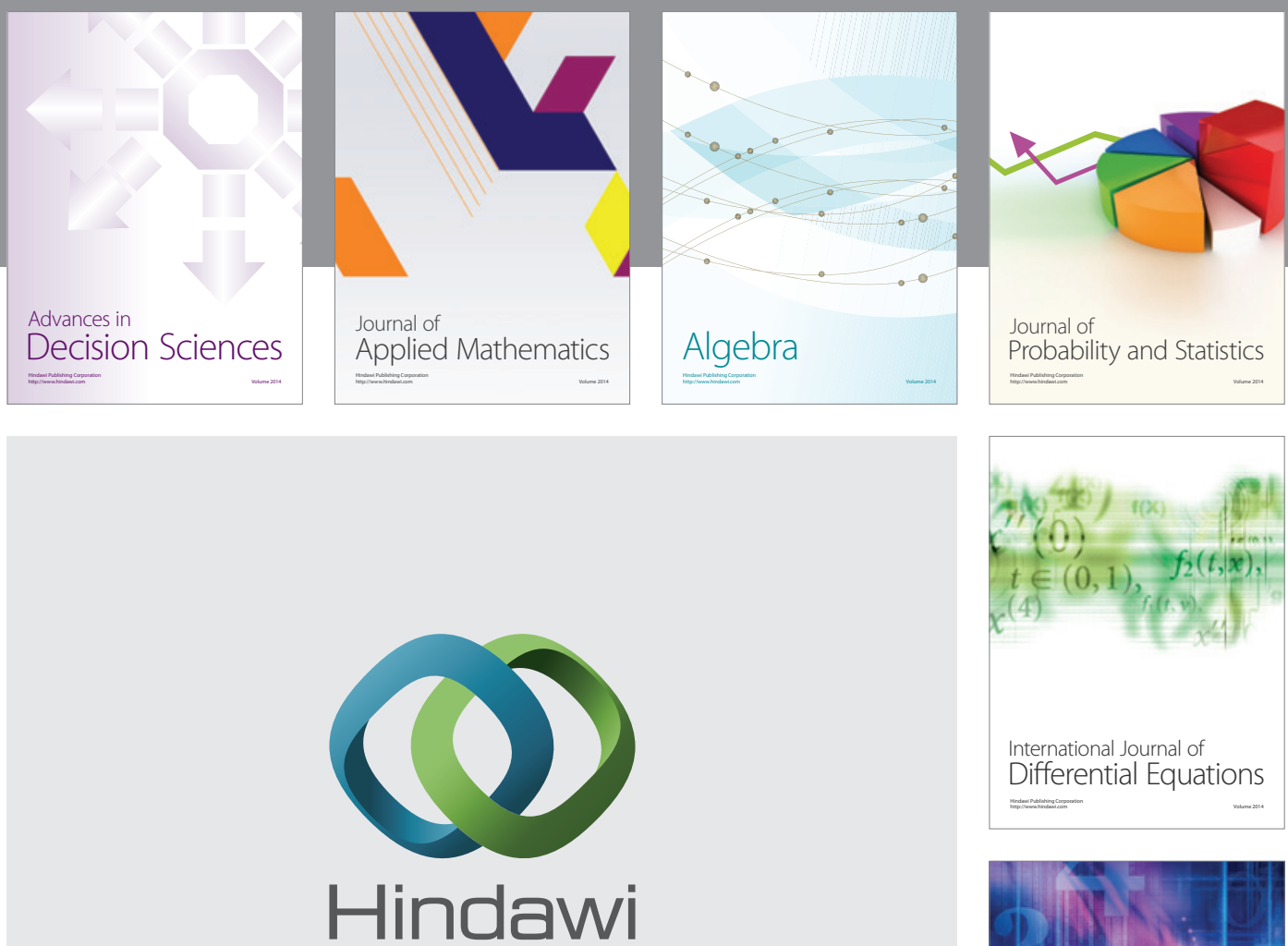

Submit your manuscripts at http://www.hindawi.com
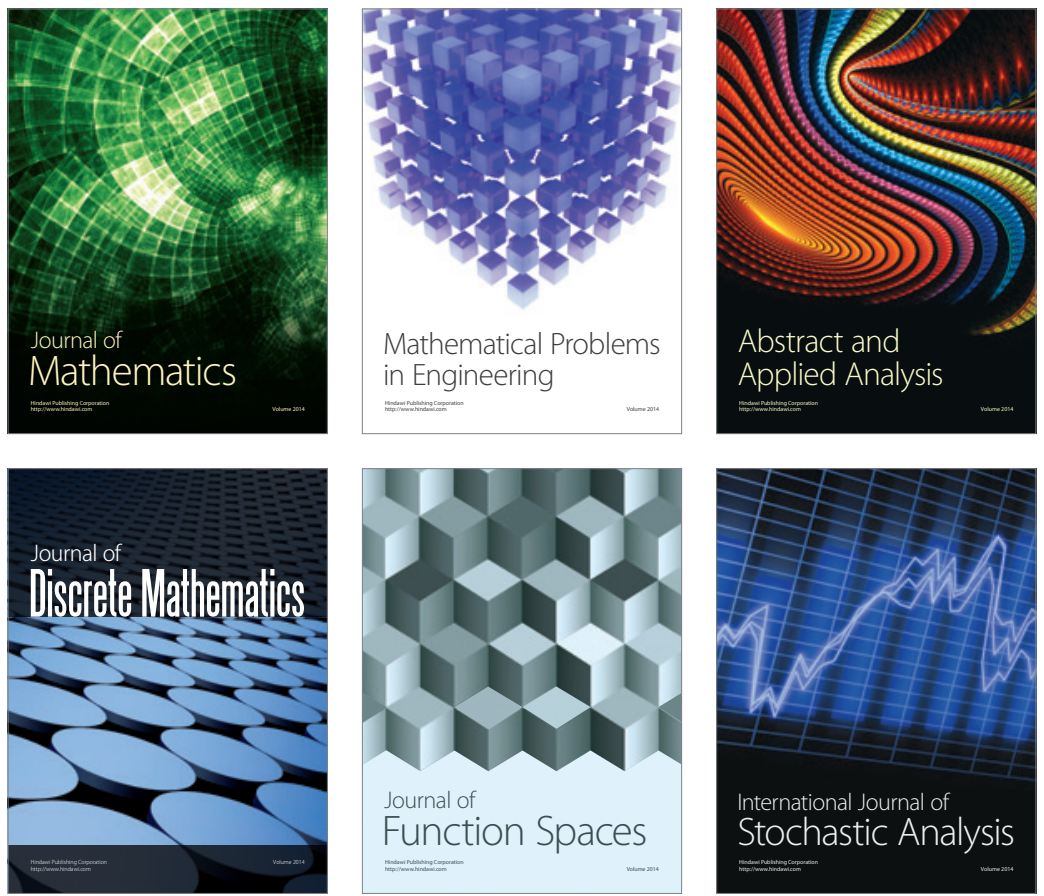

Journal of

Function Spaces

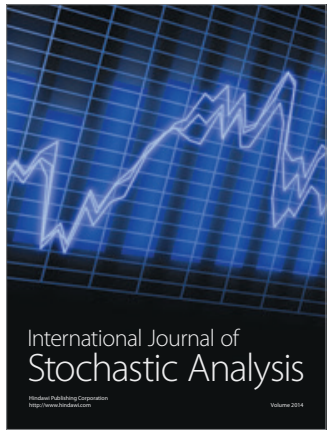

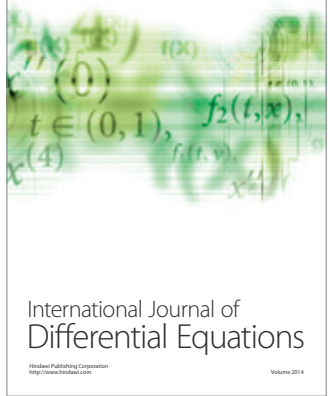
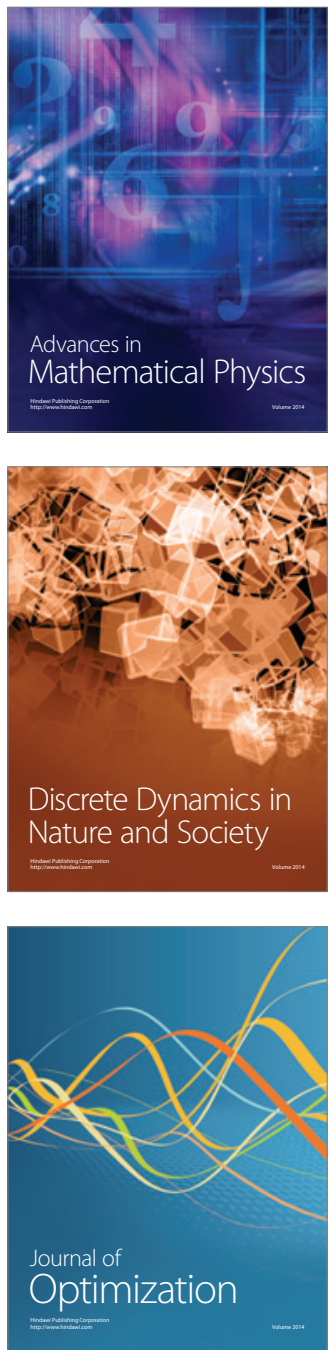